\title{
Myocardial T1 mapping using SMART1Map: initial in vivo experience
}

\author{
Jeff A Stainsby ${ }^{1 *}$, Glenn S Slavin ${ }^{2}$ \\ From 16th Annual SCMR Scientific Sessions \\ San Francisco, CA, USA. 31 January - 3 February 2013
}

\section{Background}

Recently a single-point, saturation-recovery myocardial T1 mapping sequence (SMART1Map = Saturation Method using Adaptive Recovery Times for cardiac T1 Mapping) was presented [1]. Compared to common methods like MOLLI, SMART1Map measures true T1 instead of apparent $\mathrm{T} 1$ relaxation, is more time efficient and can track exact TI times instead of estimated ones based on the heart rate during prescription. In this work we compare SMART1Map to MOLLI in an initial cohort of volunteers.

\section{Methods}

Versions of SMART1Map and MOLLI were implemented on a GE MR450w scanner. Four healthy subjects were imaged without contrast agent and with informed consent. Single shot images were acquired at the TI times. SMART1Map acquisitions collected 5 TIs in 9 heart beats at 1,1,1,2 and 4 heart beats after saturation. MOLLI acquisitions collected 8 TIs $(2,2$ and 4 heartbeats after each inversion) in 14 heart beats. A total of 12 data sets for each sequence were collected. T1 values in an ROI in the mid-septum were calculated. Values were computed with both estimated TI times based on the heart rate at the time of scan prescription and true TI times based on pulse sequence measurements of each cardiac interval during scanning. MOLLI values were computed with and without Look-Locker (LL) correction [2]. SMART1Map values represent true, not apparent, T1 values and thus no correction was applied.

\section{Results}

Measured T1 values are summarized in Table 1 . The mean T1 from SMART1Map (1193 ms) is consistent with $\mathrm{T} 1$ values reported in the literature using singlepoint methods: $\mathrm{T} 1=1219 \mathrm{~ms}$, [3] and $\mathrm{T} 1=1175 \mathrm{~ms}$ [4]. SMART1Map acquires its two long ( $>1 R R)$ TI samples in two separate blocks of multiple ( 2 and 4 ) heartbeats so it is important to account for the effect of heart rate variations on the actual TI times to achieve robust T1 measurements. This is demonstrated by the difference in T1s when using estimated and actual TIs for SMART1Map as in Table 1. Figure 1 plots heart rate variability over all series in these subjects relative to the fixed rate at scan prescription, illustrating $20 \%$ variations in heart rate over a breath

Table 1 Mean measured T1 values in normal myocardium.

\begin{tabular}{ccc}
\hline Mean T1 values ( $\mathrm{N}=12)$ & T1 (ms) \\
\hline MOLLI no LL correction & 792.1 & 1018.2 \\
MOLLI with LL correction and measured TI times & 1012.8 \\
SMART1Map with estimated TI times & 1279.1 \\
SMART1Map with measured (true) TI times & 1193.0 \\
\hline
\end{tabular}

Three series for each sequence in each of 4 volunteers were acquired. Values measured using a version of MOLLI with no correction, with the common LookLocker correction and with the Look-Locker correction and correcting TI times based on pulse sequence-measured RR durations are listed. Values measured using SMART1Map with estimated TI times and the corrected TI times are also listed. The T1 values from SMART1Map with measured TI times closely match unenhanced myocardium T1's measured with single-point methods in the literature.

${ }^{1}$ GE Healthcare, Toronto, ON, Canada

Full list of author information is available at the end of the article

() Biomed Central

(c) 2013 Stainsby and Slavin; licensee BioMed Central Ltd. This is an Open Access article distributed under the terms of the Creative Commons Attribution License (http://creativecommons.org/licenses/by/2.0), which permits unrestricted use, distribution, and reproduction in any medium, provided the original work is properly cited. 


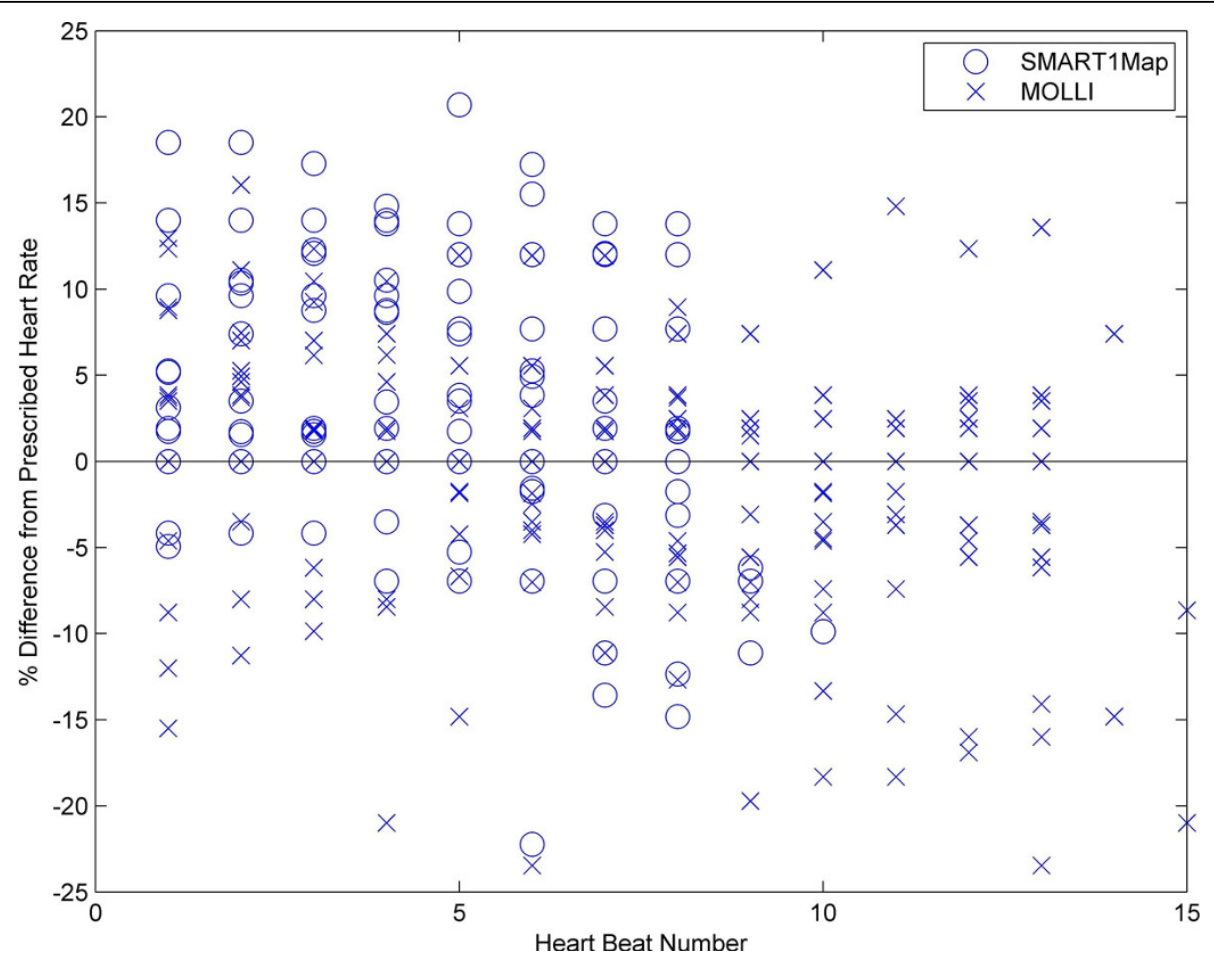

Figure 1 For all acquisitions the pulse sequence recorded in real-time the actual duration of each RR interval during scanning from which an instantaneous heart rate is determined. The variation in this beat-by-beat heart rate as a percentage of the fixed heart rate determined at the scan prescription stage is plotted as a function of heart beat number during the scan. Data for SMART1Map (o's) and MOLLI (x's) are shown and demonstrate heart rate variations can reach $20 \%$ across even these short breath-held scans.

hold were seen. MOLLI demonstrates less benefit from the use of actual TI times, resulting in T1 values that remain underestimated relative to the expected T1s. This may be due to a variety of confounding factors including the interleaving of TIs, the influence of differential signal recovery, and the behavior of the LL correction, which is not clearly understood and may obscure the effects of heart rate variation.

\section{Conclusions}

SMART1Map is a new T1 mapping method that provides accurate, breath held, true myocardial $\mathrm{T} 1$ values for normal myocardium. Compared to literature values, it provides more accurate $\mathrm{T} 1$ measurements compared to MOLLI which studies have shown underestimates long T1s.

\section{Funding}

N/A

Author details

${ }^{1} \mathrm{GE}$ Healthcare, Toronto, ON, Canada. ${ }^{2} \mathrm{GE}$ Healthcare, Bethesda, MD, USA.

Published: 30 January 2013

\section{References}

1. Slavin, et al:. Proc ISMRM 2012.

2. Deichmann, et al.. JMR 1992.

3. Wacker, et al:: MRM 1999.

4. Chow, et al.. Proc ISMRM 2012

doi:10.1186/1532-429X-15-S1-P13

Cite this article as: Stainsby and Slavin: Myocardial T1 mapping using SMART1Map: initial in vivo experience. Journal of Cardiovascular Magnetic Resonance 2013 15(Suppl 1):P13.
Submit your next manuscript to BioMed Central and take full advantage of:

- Convenient online submission

- Thorough peer review

- No space constraints or color figure charges

- Immediate publication on acceptance

- Inclusion in PubMed, CAS, Scopus and Google Scholar

- Research which is freely available for redistribution

Submit your manuscript at www.biomedcentral.com/submit 